\title{
An Unusual Metastatic Renal Cell Carcinoma with Maintained Complete Response to Sunitinib Treatment
}

\author{
Luis Chara $^{a} \quad$ Beatriz Rodríguez $^{\mathrm{b}} \quad$ Esther Holgado $^{\mathrm{a}}$ \\ Natalia Ramírez ${ }^{a} \quad$ Isabel Fernández-Rañada ${ }^{a}$ \\ Nicolás Mohedano ${ }^{a} \quad$ Alberto Arcediano $^{a}$ Inés García ${ }^{a}$ \\ Javier Cassinello ${ }^{a}$ \\ ${ }^{a}$ Medical Oncology Service and ${ }^{b}$ Pharmacy Service, Guadalajara University \\ General Hospital, Guadalajara, Spain
}

\section{Key Words}

Renal cell carcinoma - Thyroid metastases - Duodenal metastases .

Duodenopancreatectomy · Sunitinib · Complete response $\cdot$ Long-term survivorship

\begin{abstract}
Recently, metastatic renal cell carcinoma (mRCC) treatment has changed dramatically with the onset of new therapies against molecular targets replacing immunotherapy as standard treatment. We report the case of a 49-year-old patient with a moderately differentiated renal clear cell carcinoma without extracapsular extension who underwent radical nephrectomy. Eight months after surgery, he developed a thyroid metastasis which was also treated surgically with a hemithyroidectomy. Seventy-five months after nephrectomy, the patient presented an upper gastrointestinal bleeding due to a duodenal metastasis that infiltrates the head of the pancreas. The treatment applied was surgery by duodenopancreatectomy, with positive surgical margins in the pathologic study. In addition to this, the extension study showed lung metastases requiring initiation of systemic treatment with sunitinib. The patient presented an excellent response to treatment, showing complete clinical and radiological response at 5 months of treatment (RECIST criteria) and a disease-free survival of 48 months until now, without evidence of toxicity. RCC has the potential to metastasize to almost any location, but thyroid and duodenal metastases in RCC are extremely rare. Moreover, this case also highlights the good responses that can be achieved in terms of disease-free survival, low toxicity and quality of life in this new era of therapies against molecular targets.
\end{abstract}




\section{Introduction}

Renal cell carcinoma (RCC) represents approximately $85 \%$ of all renal tumors [1]. Initial treatment for localized disease is surgical, but between 20 and $30 \%$ of patients relapse with a mean time to progression of 1-2 years. Approximately one third of patients present with metastatic disease with an overall survival of 12-15 months [2].

Few years ago, metastatic RCC (mRCC) patients had few treatment options. Cytokines like interleukin-2 (IL-2) and interferon- $\alpha$ (IFN- $\alpha$ ) were the standard treatment, but were associated with low response rates and significant toxicity.

In the last 5 years, mRCC treatment has changed dramatically, with the onset of new therapies against molecular targets such as the vascular-endothelial growth factor receptor (VEGFR) and mTOR signalling pathway. Both treatments in both first and second line have shown clear benefits in these patients and have replaced immunotherapy as standard treatment $[3,4]$.

\section{Case Report}

A 49-year-old male with a personal history of arterial hypertension and smoking was referred to the urology department due to a history of monosymptomatic macroscopic hematuria. The initial ultrasonography study demonstrated an $8.5-\mathrm{cm}$ solid mass with a central necrotic area at the lower pole of the left kidney, compatible with RCC. The computed tomography (CT) scan confirmed the presence of a renal tumor that invades the renal sinus, but without vascular or regional node compromise or distant metastases. Radical nephrectomy was then performed with the pathological diagnosis of a moderately differentiated renal clear cell carcinoma with absence of extracapsular or vascular extension $\left(\mathrm{T}_{2 \mathrm{a}} \mathrm{N}_{0} \mathrm{M}_{0}\right)$.

Without postoperative complications, the patient continued routine follow-up visits every 3 months in the urology service. Eight months after nephrectomy, while the patient was asymptomatic, a control CT demonstrated a 4-cm thyroid nodule in the left lobe without any other finding. The patient was then referred to endocrinology where the study was completed with a thyroid scintigraphy that confirmed the cold thyroid nodule diagnosis. Thyroid function tests were normal and fine needle aspiration cytology was performed, with the pathological result compatible with follicular proliferation. Nevertheless, left hemithyroidectomy was carried out with the pathological result of renal clear cell carcinoma metastases associated with a thyroid nodular hyperplasia. The CT performed after surgical intervention revealed no other compromised organs or metastatic extension.

Subsequently, the patient was referred to our medical oncology service where routine follow-up visits were carried out keeping an expectant attitude since the CT scan, physical examination and lab tests performed routinely were normal.

Seventy-five months after nephrectomy (67 months after the first metastases), the patient presented at the emergency department with a history of one episode of melena that required his admission to the gastroenterology service where the endoscopic study revealed an upper gastrointestinal bleeding due to a duodenal ulcer (not biopsied) that required sclerotherapy treatment. Helicobacter pylori $(\mathrm{Hp})$ test was positive and the patient received eradication treatment with subsequent negativization of breath test.

However, 5 months after the first episode of duodenal bleeding, the patient was admitted again to the gastroenterology service due to another upper gastrointestinal bleeding episode, this time associated with fatigue. The lab test was significant for microcytic hypochromic anemia with hemoglobin of $5.3 \mathrm{~g} / \mathrm{dl}$, requiring transfusion of 3 erythrocyte concentrates and bowel rest. The gastroscopy again revealed a duodenal ulcer (biopsy was taken at that time) corresponding to the same lesion detected 5 months earlier without signs of healing despite sclerotherapy and $H p$ eradication treatment. Because of this and the big size of ulcer, an abdominal CT scan was performed 
showing the presence of enlargement of the head of the pancreas without any other finding. The study was completed with an ultrasound endoscopy that confirmed the presence of a 4-cm solid mass in the head of the pancreas with 'neoplastic aspect' that infiltrated the adjacent duodenal wall. The pathologic result confirmed the presence of metastatic infiltration of renal clear cell carcinoma in duodenal mucosa. For that reason, it was decided to carry out a duodenopancreatectomy in which the pathological study showed the presence of multiple metastatic invasion of renal clear cell carcinoma in pancreas and duodenal wall with positive surgical margins. After recovery from a postoperative period complicated with diabetes mellitus and steatorrhea, a new control CT scan was performed in April 2007 (82 months after nephrectomy) showing the presence of a 1.4-cm left lung nodule

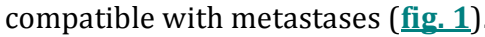

Given the presence of lung metastases and positive surgical margins in duodenopancreatectomy, it was decided to start first-line systemic treatment with sunitinib $50 \mathrm{mg}$ once a day for 4 weeks with 2 weeks rest) in May 2007. After 5 months of treatment, the patient showed complete response with disappearance of lung metastases in the control CT scan as well as absence of metastatic disease in other organs without toxicity (fig. 1).

Currently, after 48 months of follow-up (132 months after nephrectomy), the patient continues treatment with sunitinib at the same dose, maintaining complete response in regular control CT studies (RECIST criteria), with good tolerance to treatment except for an episode of fever and moderate asthenia that has not required treatment adjustment, and maintaining an excellent performance status (ECOG 0) at all times.

\section{Discussion}

RCC has the potential to metastasize to almost any location, the most common sites being lung, lymph nodes, liver, bone, adrenal glands and brain.

Thyroid metastases in RCC are extremely rare, representing less than 5\% of cases, most of which are metachronous, with a mean latency period of about 10 years. The usual clinical presentation is of a thyroid nodule or goiter with normal thyroid function tests, which in the scintigraphy behaves like cold nodules. Our patient presented with left thyroid lobe metastases 8 months after nephrectomy that required hemithyroidectomy as treatment. The clinical presentation was similar to data reported in the literature, emphasizing the importance of raised suspicion of metastases in all patients with a history of RCC and thyroid volume increase [5].

Likewise, only 28 cases of duodenal metastases from RCC have been described, thus turning it into a rare entity. The vast majority of cases described presented upper gastrointestinal bleeding, but may also show anemia, fatigue, bowel obstruction or jaundice. The mean time of presentation after nephrectomy is approximately $7.9 \pm 4.7$ years, and the most common form of treatment is surgical resection by duodenopancreatectomy, besides other therapeutic options such as embolization, palliative radiotherapy or palliative gastrojejunostomy [6]. Our patient presented with duodenal metastases that compromised the head of the pancreas, and presenting with upper gastrointestinal bleeding. The treatment applied in this case was surgery by duodenopancreatectomy, with the inconvenience that the pathology result detected positive surgical margins and also that the extension study showed lung metastases requiring initiation of systemic treatment.

Sunitinib is a multitargeted tyrosine kinase inhibitor that predominantly targets VEGF activity. It was approved by the FDA (Food and Drug Administration) and EMA (European Medicines Agency) for the treatment of mRCC since 2007, thanks to the 
results obtained in a randomized, double-blind, multicenter phase III study that compared single-agent sunitinib versus IFN- $\alpha$ in first-line treatment. This study demonstrates the superiority of sunitinib vs. IFN- $\alpha$, with a mean disease-free survival of 11 vs. 5 months, a response rate of 31 vs. $6 \%$, and an overall survival of 28 vs. 14 months $[3,7]$. Our patient presented an excellent response to sunitinib treatment, showing complete clinical and radiological response at 5 months of treatment (RECIST criteria) and a disease-free survival of 48 months until now, without evidence of toxicity, and with an excellent performance status that allows him to work and develop his life normally.

This case report highlights the importance of close follow-up and the raised suspicion level that we must keep in nephrectomized RCC patients at the onset of new symptoms. Moreover, this case also highlights the good responses that can be achieved in terms of disease-free survival, low toxicity and quality of life in this new era of therapies against molecular targets; even, as in our case, better results than those described in the literature.
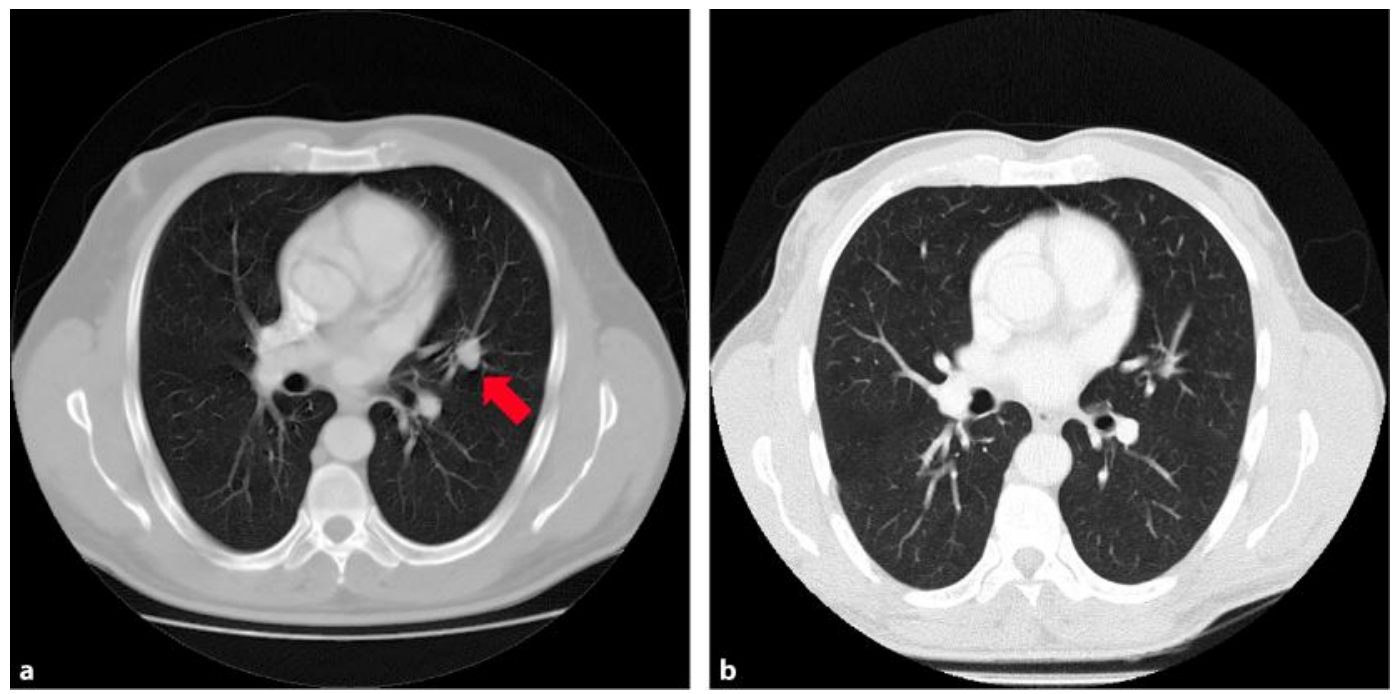

Fig. 1. Computed tomography scan of the chest showing a metastatic nodule in left lung before systemic treatment with Sunitinib (a), and complete response after 5 months of treatment (b).

\section{References}

1 Motzer RJ: New perspectives on the treatment of metastatic renal cell carcinoma: an introduction and historical overview. Oncologist 2011;16(suppl 2):1-3.

-2 Jemal A, Siegel R, Xu J, Ward E: Cancer statistics, 2010. CA Cancer J Clin 2010;60:277-300.

-3 Motzer RJ, Hutson TE, Tomczak P, et al: Sunitinib versus interferon alfa in metastatic renal-cell carcinoma. N Engl J Med 2007;356:115-124.

4 Powles T, Chowdhury S, Jones R, et al: Sunitinib and other targeted therapies for renal cell carcinoma. $\mathrm{Br}$ J Cancer 2011;104:741-745.

5 Garcia-Olaverri Rodriguez J, Villafruela Mateo A, Azurmendi Arin I, et al: [Metachronic thyroid metastasis secondary to renal carcinoma. Case report]. Arch Esp Urol 2007;60:697-699.

-6 Rustagi T, Rangasamy P, Versland M: Duodenal bleeding from metastatic renal cell carcinoma. Case Rep Gastroenterol 2011;5:249-257.

$\rightarrow 7$ Hutson TE: Targeted therapies for the treatment of metastatic renal cell carcinoma: clinical evidence. Oncologist 2011;16(suppl 2):14-22. 